Mitio Takano

\title{
A MODIFIED SUBFORMULA PROPERTY FOR THE MODAL LOGIC S4.2
}

\begin{abstract}
The modal logic $\mathrm{S} 4.2$ is $\mathrm{S} 4$ with the additional axiom $\diamond \square A \supset \square \diamond A$. In this article, the sequent calculus GS4.2 for this logic is presented, and by imposing an appropriate restriction on the application of the cut-rule, it is shown that, every GS4.2-provable sequent $S$ has a GS4.2-proof such that every formula occurring in it is either a subformula of some formula in $S$, or the formula $\square \neg \square B$ or $\neg \square B$, where $\square B$ occurs in the scope of some occurrence of $\square$ in some formula of $S$. These are just the K5-subformulas of some formula in $S$ which were introduced by us to show the modified subformula property for the modal logics K5 and K5D (Bull Sect Logic 30: 115-122, 2001). Some corollaries including the interpolation property for S4.2 follow from this. By slightly modifying the proof, the finite model property also follows.
\end{abstract}

Keywords: modal logic S4.2, sequent calculus, subformula property.

\section{Introduction}

The modal logic $\mathrm{S} 4.2$ is $\mathrm{S} 4$ with the additional axiom $\diamond \square A \supset \square \diamond A$, and it is characterized by the class of the Kripke frames whose accessibility relation $R$ is reflexive, transitive and convergent (If $u R v$ and $u R w$, then $v R x$ and $w R x$ for some $x$.). See Hughes-Cresswell [2, p. 134], for example. In this article, the sequent calculus GS4.2 for this logic is presented, and by imposing an appropriate restriction on the application of the cut-rule, it is shown that, every GS4.2-provable sequent $S$ has a GS4.2-proof such that every formula occurring in it is either a subformula of some formula in 
$S$, or the formula $\square \neg \square B$ or $\neg \square B$, where $\square B$ occurs in the scope of some occurrence of $\square$ in some formula of $S$. These are just the K5-subformulas of some formula in $S$ which were introduced by us to show the modified subformula property for the modal logics K5 and K5D (Takano [3]).

By slightly modifying the proof, the finite model property for S4.2 follows. The interpolation property for S4.2 also follows by the so-called "Maehara method" (cf. Takeuti [5]); as a by-product, one obtains Halldén completeness of S4.2: If $A \vee B$ is provable in $\mathrm{S} 4.2$, and if no propositional letter occurs in $A$ and $B$ in common, then $A$ or $B$ is provable. Moreover, by inspection of the proof of the 'only if' part of Proposition 2.1 below, an S4.2-version of Fitting's subformula results can be seen (Fitting [1]): If $A$ is provable in $\mathrm{S} 4.2$, it is obtained by zero or more applications of modus ponens and necessitation from theorems of $\mathrm{S} 4$ and formulas of the form $\diamond \square \neg \square B \supset \square \diamond \neg \square B$ where $\square B$ occurs in the scope of some occurrence of $\square$ in $A$.

In this paper, only $\neg$ (negation), $\supset$ (implication) and $\square$ (necessity) are used as the logical symbols, and others are considered as abbreviations for simplicity; thus for example, $\diamond$ abbreviates $\neg \square \neg$. Propositional letters and formulas are denoted by $p, q, r, \ldots$ and $A, B, C, \ldots$, respectively. A sequent is an expression of the form $\Gamma \rightarrow \Theta$, where the antecedent $\Gamma$ and the succedent $\Theta$ are finite sequences of formulas. But, for convenience, the antecedent and succedent of the sequent are recognized as sets also. Finite sequences (as well as finite sets) of formulas are denoted by $\Gamma, \Theta, \Delta, \Lambda, \ldots$. We mean by $\square \Gamma$ the set $\{\square A \mid A \in \Gamma\}$, and similarly for $\neg \square \Gamma$ and $\square \neg \square \Gamma$. In describing formal proofs in sequent calculi, applications of the structural rules except the cut-rule are neglected, and consecutive applications of logical rules to one formula are often combined into one.

For sequent calculus, consult Takeuti [5], for example.

\section{The sequent calculus GS4.2}

It is well-known that the modal logic $\mathrm{S} 4$ is formulated as the sequent calculus, say GS4, which is obtained from the calculus LK for the classical propositional logic by adding the following two inference rules, and it is also known that GS4 admits cut-elimination:

$$
(\square \rightarrow) \frac{A, \Gamma \rightarrow \Theta}{\square A, \Gamma \rightarrow \Theta} \quad(\rightarrow \square)_{\mathrm{S} 4} \frac{\square \Gamma \rightarrow A}{\square \Gamma \rightarrow \square A}
$$


Our sequent calculus GS4.2 for S4.2 is obtained from GS4 by extending $(\rightarrow \square)_{\mathrm{S} 4}$ to the following one:

$$
(\rightarrow \square)_{\mathrm{S} 4.2} \frac{\square \Gamma \rightarrow \square \neg \square \Theta, A}{\square \Gamma \rightarrow \square \neg \square \Theta, \square A}
$$

By the following proposition, GS4.2 is really a sequent calculus for S4.2, that is, a sequent $\Gamma \rightarrow \Theta$ is GS4.2-provable iff the corresponding formula $\bigwedge \Gamma \supset \bigvee \Theta$ is provable in S4.2.

Proposition 2.1. A sequent is GS4.2-provable iff it is GS4G-provable, where the latter is GS4 with the additional initial sequent of the form $\diamond \square A \rightarrow \square \diamond A$.

Proof: The 'if' part: It suffices to show that the additional initial sequent $\diamond \square A \rightarrow \square \diamond A$ is GS4.2-provable:

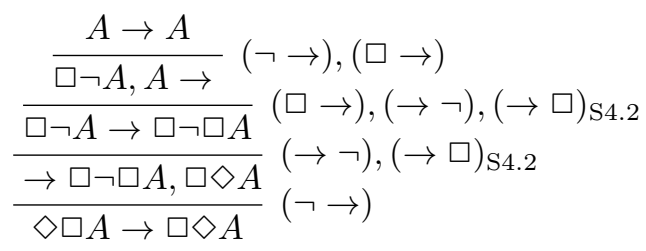

The 'only if' part: It suffices to show that GS4G-provability of the upper sequent $\square \Gamma \rightarrow \square \neg \square \Theta, A$ of the rule $(\rightarrow \square)_{\mathrm{S} 4.2}$ implies that of the lower sequent $\square \Gamma \rightarrow \square \neg \square \Theta, \square A$. First, two GS4G-proofs are given.

$$
\begin{gathered}
\vdots \text { GS4G-proof } \\
\frac{\square \Gamma \rightarrow \square \neg \square \Theta, A}{\square \neg \square \neg \square \Theta, \square \Gamma \rightarrow A}(\neg \rightarrow) \text { 's, }(\square \rightarrow) \text { 's } \\
\frac{\square \neg \square \neg \square \Theta, \square \Gamma \rightarrow \square A}{\square \Gamma \rightarrow \square A, \diamond \square \neg \square \Theta}(\rightarrow \neg) \text { S4 }
\end{gathered}
$$

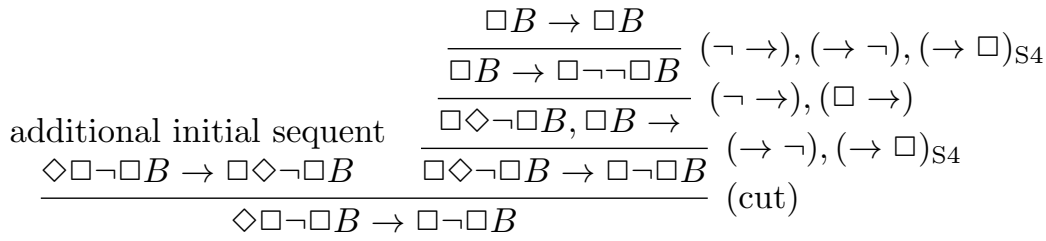

Then, by applying (cut)'s to $\square \Gamma \rightarrow \square A, \diamond \square \neg \square \Theta$ and $\diamond \square \neg \square B \rightarrow$ $\square \neg \square B$ for each $B \in \Theta$, the sequent $\square \Gamma \rightarrow \square \neg \square \Theta, \square A$ is obtained. 
But regrettably, our calculus GS4.2 neither admits cut-elimination nor enjoys the subformula property. For example, the sequent

$$
S: \rightarrow \square \neg \neg \neg \square \neg p, \square \neg \neg \neg \square p
$$

is GS4.2-provable by applying (cut) to the following GS4.2-proofs:

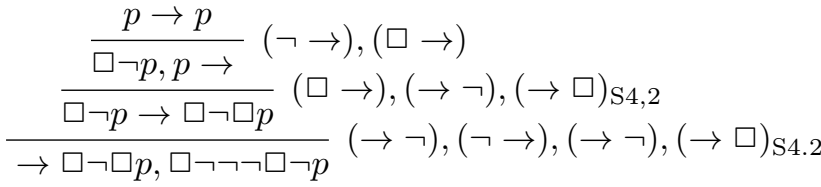

$$
\begin{aligned}
& \begin{array}{c}
\frac{\square p \rightarrow \square p}{\square \neg \square p, \square p \rightarrow}(\neg \rightarrow),(\square \rightarrow) \\
\square \neg \square p \rightarrow \square \neg \neg \neg \square p
\end{array}(\rightarrow \neg),(\neg \rightarrow),(\rightarrow \neg),(\rightarrow \square)_{\mathrm{S} 4.2}
\end{aligned}
$$

But $S$ has neither cut-free GS4.2-proof nor GS4.2-proof consisting solely of subformulas of either formula in $S$. For, since the concatenation $\square \neg \square$ does not occur in $S$, any GS4.2-proof of $S$ of those forms must be a GS4proof in reality, which is a contradiction.

So, we will modify the notion of subformula.

Definition 2.2 ([3, Definition 1]). (1) An internal subformula of $A$ is a subformula of some formula $C$ such that $\square C$ is a subformula of $A$.

(2) A K5-subformula of $A$ is either a subformula of $A$ or the formula of the form $\square \neg \square B$ or $\neg \square B$, where $\square B$ is an internal subformula of $A$.

The sets of all the subformulas, internal subformulas and K5-subformulas of some formulas in $\Gamma$ are denoted by $\operatorname{Sf}(\Gamma), \operatorname{InSf}(\Gamma)$ and $\operatorname{Sf}_{\mathrm{K} 5}(\Gamma)$, respectively.

If $\square A$ is an internal subformula of $B$, and $B$ is a $\mathrm{K} 5$-subformula of $C$, then $\square A$ is an internal subformula of $C$. If $A$ is a K5-subformula of $B$, and $B$ is a K5-subformula of $C$, then $A$ is a K5-subformula of $C$.

THEOREM 2.3. Every GS4.2-provable sequent $\Gamma \rightarrow \Theta$ has a GS4.2-proof such that every formula occurring in it belongs to $\operatorname{Sf}_{\mathrm{K} 5}(\Gamma \cup \Theta)$.

To show this, the cut-rule is restricted to the following one:

$$
\begin{gathered}
\text { (cut) })_{\mathrm{K} 5} \frac{\Gamma \rightarrow \Theta, \square \neg \square A \quad \square \neg \square A, \Delta \rightarrow \Lambda}{\Gamma, \Delta \rightarrow \Theta, \Lambda}, \\
\text { where } \square A \in \operatorname{InSf}(\Gamma \cup \Theta \cup \Delta \cup \Lambda) .
\end{gathered}
$$


Let's call this restricted GS4.2 as GS4.2- ${ }^{-}$It is clear that every formula occurring in the upper sequents of (cut) $\mathrm{K5}$ is a $\mathrm{K} 5$-subformula of some formula occurring in the lower one, and so every formula occurring in a GS4. $2^{-}$-proof is a K5-subformula of some formula occurring in the endsequent.

So, it suffices to show the following lemma for the proof of Theorem 2.3. Lemma 2.4. Every GS4.2-provable sequent is GS4.2--provable .

We will show this lemma in the next section.

\section{Proof of Lemma 2.4}

We will prove the contraposition of this lemma by constructing the universal Kripke model $\langle W, R, V\rangle$ for $\mathrm{S} 4.2$ such that every GS4.2- ${ }^{-}$-unprovable sequent is rejected in some point in $W$.

Definition 3.1. A sequent $\Delta \rightarrow \Lambda$ is downward saturated, iff it is GS4.2unprovable and the following properties hold for every $A$ and $B$ :

(3.1-a) If $\neg A \in \Delta$ then $A \in \Lambda$.

(3.1-b) If $\neg A \in \Lambda$ then $A \in \Delta$.

(3.1-c) If $A \supset B \in \Delta$ then either $A \in \Lambda$ or $B \in \Delta$.

(3.1-d) If $A \supset B \in \Lambda$ then $A \in \Delta$ and $B \in \Lambda$.

(3.1-e) If $\square A \in \Delta$ then $A \in \Delta$.

Downward saturated sequents are denoted by $u, v, w, x, \ldots$; besides, $\mathrm{a}(u)$ and $\mathrm{s}(u)$ denote the antecedent and succedent of $u$, respectively.

Thanks to the initial sequents of LK, $\mathrm{a}(u) \cap \mathrm{s}(u)=\emptyset$ for every $u$.

It is routine to show the following proposition.

Proposition 3.2. If $\Gamma \rightarrow \Theta$ is GS4.2- $2^{-}$unprovable, then $\Gamma \subseteq \mathrm{a}(u), \Theta \subseteq$ $\mathrm{s}(u)$ and $\mathrm{a}(u) \cup \mathrm{s}(u) \subseteq \operatorname{Sf}(\Gamma \cup \Theta)$ for some downward saturated sequent $u$.

Definition 3.3 (The canonical model $\langle W, R, V\rangle$ ). (1) $W$ is the set of all the downward saturated sequents $u$ 's that satisfy the following property $W(u)$ :

For every $B$, if $\square B \in \operatorname{InSf}(\mathrm{a}(u) \cup \mathrm{s}(u))$ then $\square \neg \square B \in \mathrm{a}(u) \cup \mathrm{s}(u)$.

(2) The binary relation $R$ on the downward saturated sequents is defined by:

$$
u R v, \text { iff } u R_{\mathrm{S} 4} v, u R_{\mathrm{S} 4.2}^{\prime} v, u Q v \text { and } v R_{\mathrm{S} 4.2}^{\prime} u,
$$


where

- $u R_{\mathrm{S} 4} v$, iff $\square B \in \mathrm{a}(u)$ implies $\square B \in \mathrm{a}(v)$ for every $B$;

- $u R_{\mathrm{S} 4.2}^{\prime} v$, iff $\square \neg \square B \in \mathrm{s}(u)$ implies $\square \neg \square B \in \mathrm{s}(v)$ for every $B$; and

- $u Q v$, iff $\square B \in \mathrm{a}(v)$ implies either $\square B \in \mathrm{a}(u)$ or $\square \neg \square B \in \mathrm{s}(u)$ for every $B$.

The relation $R$ restricted to $W \times W$ is also denoted by $R$.

(3) $V$ is the function of the propositional letters to the subsets of $W$ such that $V(p)=\{u \in W \mid p \in \mathrm{a}(u)\}$ for every $p$.

REMARK 3.4. For a GS4.2-unprovable sequent $\Gamma \rightarrow \Theta$, if $W$ is restricted to those $u$ 's such that $\mathrm{a}(u) \cup \mathrm{s}(u) \subseteq \mathrm{Sf}_{\mathrm{K} 5}(\Gamma \cup \Theta)$, the following argument remains valid. So, the finite model property for $\mathrm{S} 4.2$ follows, since the restricted $W$ is a finite set.

Proposition 3.5. The relation $R$ on $W$ is reflexive and transitive.

Proof: Reflexiveness is evident. For the proof of transitivity, suppose $u R v$ and $v R w$. To conclude $u R w$, the four properties $u R_{\mathrm{S} 4} w, u R_{\mathrm{S} 4.2}^{\prime} w, u Q w$ and $w R_{\mathrm{S} 4.2}^{\prime} u$ must be checked, but those other than $u Q w$ are clear by the transitivity of $R_{\mathrm{S} 4}$ and $R_{\mathrm{S} 4.2}^{\prime}$. So to show $u Q w$, suppose $\square B \in \mathrm{a}(w)$. By $v Q w$, either $\square B \in \mathrm{a}(v)$ or $\square \neg \square B \in \mathrm{s}(v)$. In the former case, $\square B \in \mathrm{a}(u)$ or $\square \neg \square B \in \mathrm{s}(u)$ by $u Q v$. In the latter case, $\square \neg \square B \in \mathrm{s}(u)$ by $v R_{\mathrm{S} 4.2}^{\prime} u$.

To save the similar argument in the proofs of Propositions 3.7 and 3.10, the following short remark is made.

REMARK 3.6. If $u R_{\mathrm{S} 4} v$ and $\square B \in \mathrm{a}(v)$, then $\square \neg \square B \notin \mathrm{a}(u)$. For, if $\square \neg \square B$ were in a $(u)$, it would also be in a $(v)$ by $u R_{\mathrm{S} 4} v$, but this is a contradiction, since the sequent $\square \neg \square B, \square B \rightarrow$ is provable by applying $(\neg \rightarrow)$ and $(\square \rightarrow)$ successively to $\square B \rightarrow \square B$, and so it is not the case that both $\square \neg \square B$ and $\square B$ are in $\mathrm{a}(v)$.

Proposition 3.7. For every $u \in W$, there is a $u^{\#} \in W$ with the following property:

(3.7-a) If $u R v$ then $v R u^{\#}$ for every $v \in W$.

Proof: Given $u \in W$, put $\Gamma=\{B \mid \square B \in \mathrm{a}(u)\}$ and $\Theta=\{B \mid$ $\square \neg \square B \in \mathrm{s}(u)\}$. Then the sequent $\square \Theta, \square \Gamma \rightarrow \square \neg \square \Theta$ is unprovable; for, if it were provable, $\square \Gamma \rightarrow \square \neg \square \Theta$ would become provable by $(\rightarrow \neg)$ 's and $(\rightarrow \square)_{\mathrm{S} 4.2}$ 's, which is a contradiction. So by Proposition 3.2, $\square \Theta \subseteq \mathrm{a}\left(u^{\#}\right)$, $\square \Gamma \subseteq \mathrm{a}\left(u^{\#}\right), \square \neg \square \Theta \subseteq \mathrm{s}\left(u^{\#}\right)$ and $\mathrm{a}\left(u^{\#}\right) \cup \mathrm{s}\left(u^{\#}\right) \subseteq \operatorname{Sf}(\square \Theta \cup \square \Gamma \cup \square \neg \square \Theta)$ 
for some downward saturated sequent $u^{\#}$. It follows $u R_{\mathrm{S} 4} u^{\#}$ and $u R_{\mathrm{S} 4.2}^{\prime} u^{\#}$ from $\square \Gamma \subseteq \mathrm{a}\left(u^{\#}\right)$ and $\square \neg \square \Theta \subseteq \mathrm{s}\left(u^{\#}\right)$, respectively.

Let's show $W\left(u^{\#}\right)$ to testify $u^{\#} \in W$. So suppose $\square B \in \operatorname{InSf}\left(\mathrm{a}\left(u^{\#}\right) \cup\right.$ $\left.\mathrm{s}\left(u^{\#}\right)\right)$. Since $\mathrm{a}\left(u^{\#}\right) \cup \mathrm{s}\left(u^{\#}\right) \subseteq \operatorname{Sf}(\square \Theta \cup \square \Gamma \cup \square \neg \square \Theta) \subseteq \operatorname{Sf}(\mathrm{a}(u) \cup \mathrm{s}(u))$, it follows $\square B \in \operatorname{InSf}(\mathrm{a}(u) \cup \mathrm{s}(u))$, so $\square \neg \square B \in \mathrm{a}(u) \cup \mathrm{s}(u)$ by $W(u)$, and so $\square \neg \square B \in \mathrm{a}\left(u^{\#}\right) \cup \mathrm{s}\left(u^{\#}\right)$ by $u R_{\mathrm{S} 4} u^{\#}$ and $u R_{\mathrm{S} 4.2}^{\prime} u^{\#}$.

Next show the crucial property $(3.7-\mathrm{a})$ of $u^{\#}$. Suppose $u R v$, where $v \in W$. To conclude $v R u^{\#}$, the four properties $v R_{\mathrm{S} 4} u^{\#}, v R_{\mathrm{S} 4.2}^{\prime} u^{\#}, v Q u^{\#}$ and $u^{\#} R_{\mathrm{S} 4.2}^{\prime} v$ must be checked. We will show these by turns.

First, to show $v R_{\mathrm{S} 4} u^{\#}$, suppose $\square B \in \mathrm{a}(v)$. By $u Q v$, either $\square B \in \mathrm{a}(u)$ or $\square \neg \square B \in \mathrm{s}(u)$. In the former case, $\square B \in \mathrm{a}\left(u^{\#}\right)$ by $u R_{\mathrm{S} 4} u^{\#}$. In the latter case, since $B \in \Theta$ it follows $\square B \in \square \Theta \subseteq \mathrm{a}\left(u^{\#}\right)$.

Second, $v R_{\mathrm{S} 4.2}^{\prime} u$ and $u R_{\mathrm{S} 4.2}^{\prime} u^{\#}$ together with the transitivity of $R_{\mathrm{S} 4.2}^{\prime}$ imply $v R_{\mathrm{S} 4.2}^{\prime} u^{\#}$.

Thirdly, to show $v Q u^{\#}$, suppose $\square B \in \mathrm{a}\left(u^{\#}\right)$. Since $\square B \in \mathrm{a}\left(u^{\#}\right) \subseteq$ $\operatorname{Sf}(\square \Theta \cup \square \Gamma \cup \square \neg \square \Theta)=\square \Gamma \cup \operatorname{Sf}(\Gamma \cup \square \neg \square \Theta)$ and $\square \neg \square \Theta \subseteq \mathrm{s}\left(u^{\#}\right)$, it follows $\square B \in \square \Gamma \cup \operatorname{Sf}(\Gamma \cup \square \Theta) \subseteq \square \Gamma \cup \operatorname{InSf}(\mathrm{a}(u) \cup \mathrm{s}(u))$. So, either $\square B \in \square \Gamma$ or $\square B \in \operatorname{InSf}(\mathrm{a}(u) \cup \mathrm{s}(u))$. In the former case, $\square B \in \mathrm{a}(u)$ since $B \in \Gamma$, and so $\square B \in \mathrm{a}(v)$ by $u R_{\mathrm{S} 4} v$. In the latter case, $\square \neg \square B \in \mathrm{a}(u) \cup \mathrm{s}(u)$ by $W(u)$, but $\square \neg \square B \notin \mathrm{a}(u)$ by $u R_{\mathrm{S} 4} u^{\#}, \square B \in \mathrm{a}\left(u^{\#}\right)$ and Remark 3.6. Hence $\square \neg \square B$ is in $\mathrm{s}(u)$, and so is in $\mathrm{s}(v)$ by $u R_{\mathrm{S} 4.2}^{\prime} v$.

Lastly, to show $u^{\#} R_{\mathrm{S} 4.2}^{\prime} v$, suppose $\square \neg \square B \in \mathrm{s}\left(u^{\#}\right)$. Since $\mathrm{s}\left(u^{\#}\right) \subseteq$ $\mathrm{Sf}(\mathrm{a}(u) \cup \mathrm{s}(u))$, it follows $\square B \in \operatorname{InSf}(\mathrm{a}(u) \cup \mathrm{s}(u))$, and so $\square \neg \square B \in \mathrm{a}(u) \cup \mathrm{s}(u)$ by $W(u)$. But if $\square \neg \square B$ were in a $(u)$, it would also be in a $\left(u^{\#}\right)$ by $u R_{\mathrm{S} 4} u^{\#}$, which is a contradiction. So $\square \neg \square B$ is in $\mathrm{s}(u)$, and so in $\mathrm{s}(v)$ by $u R_{\mathrm{S} 4.2}^{\prime} v$.

Corollary 3.8. The relation $R$ on $W$ is convergent. That is, for every $u, v, w \in W$, if $u R v$ and $u R w$ then $v R x$ and $w R x$ for some $x \in W$.

Proposition 3.9. If $\Gamma \rightarrow \Theta$ is GS4.2--unprovable, then $\Gamma \subseteq \mathrm{a}(u)$ and $\Theta \subseteq \mathrm{s}(u)$ for some $u \in W$.

Proof: Let $A_{1}, A_{2}, \ldots, A_{n}$ be an enumeration of all $A$ 's such that $\square A \in$ $\operatorname{InSf}(\Gamma \cup \Theta)$. Put $\Gamma_{1}=\Gamma$ and $\Theta_{1}=\Theta$. Suppose that $\Gamma_{k}$ and $\Theta_{k}$ have been defined so that $\Gamma \subseteq \Gamma_{k}, \Theta \subseteq \Theta_{k}$, but $\Gamma_{k} \rightarrow \Theta_{k}$ is unprovable $(1 \leq k \leq$ $n)$. Then, either $\Gamma_{k} \rightarrow \Theta_{k}, \square \neg \square A_{k}$ or $\square \neg \square A_{k}, \Gamma_{k} \rightarrow \Theta_{k}$ is unprovable; for, if both were provable, since $\square A_{k} \in \operatorname{InSf}(\Gamma \cup \Theta) \subseteq \operatorname{InSf}\left(\Gamma_{k} \cup \Theta_{k}\right)$, it would follow that $\Gamma_{k} \rightarrow \Theta_{k}$ is provable by (cut) ${ }_{\mathrm{K} 5}$, which contradicts our assumption. Hence, put $\Gamma_{k+1}=\Gamma_{k}$ and $\Theta_{k+1}=\Theta_{k} \cup\left\{\square \neg \square A_{k}\right\}$, or 
$\Gamma_{k+1}=\Gamma_{k} \cup\left\{\square \neg \square A_{k}\right\}$ and $\Theta_{k+1}=\Theta_{k}$ so that $\Gamma_{k+1} \rightarrow \Theta_{k+1}$ is also unprovable.

Having defined $\Gamma_{n+1}$ and $\Theta_{n+1}$, since $\Gamma_{n+1} \rightarrow \Theta_{n+1}$ is unprovable, $\Gamma_{n+1} \subseteq \mathrm{a}(u), \Theta_{n+1} \subseteq \mathrm{s}(u)$ and $\mathrm{a}(u) \cup \mathrm{s}(u) \subseteq \operatorname{Sf}\left(\Gamma_{n+1} \cup \Theta_{n+1}\right)$ for some downward saturated sequent $u$ by Proposition 3.2.

We claim that this $u$ is the required one. Since $\Gamma \subseteq \Gamma_{n+1} \subseteq \mathrm{a}(u)$ and $\Theta \subseteq \Theta_{n+1} \subseteq \mathrm{s}(u)$, it is left to check the property $W(u)$. So suppose $\square B \in \operatorname{InSf}(\mathrm{a}(u) \cup \mathrm{s}(u))$. Since $\mathrm{a}(u) \cup \mathrm{s}(u) \subseteq \operatorname{Sf}\left(\Gamma_{n+1} \cup \Theta_{n+1}\right) \subseteq \operatorname{Sf}\left(\operatorname{Sf}_{\mathrm{K} 5}(\Gamma \cup\right.$ $\Theta)) \subseteq \operatorname{Sf}_{\mathrm{K} 5}(\Gamma \cup \Theta)$, it follows $\square B \in \operatorname{InSf}(\Gamma \cup \Theta)$, so $B$ is $A_{k}$ for some $k$ $(1 \leq k \leq n)$, and so $\square \neg \square B \in \Gamma_{k+1} \cup \Theta_{k+1} \subseteq \Gamma_{n+1} \cup \Theta_{n+1} \subseteq \mathrm{a}(u) \cup \mathrm{s}(u)$.

Proposition 3.10. If $u \in W$ and $\square A \in \mathrm{s}(u)$, then $A \in \mathrm{s}(v)$ for some $v \in W$ such that $u R v$.

Proof: Put $\Gamma=\{B \mid \square B \in \mathrm{a}(u)\}$ and $\Theta=\{B \mid \square \neg \square B \in \mathrm{s}(u)\}$. Since $\square \Gamma \rightarrow \square \neg \square \Theta, \square A$ is unprovable, neither is $\square \Gamma \rightarrow \square \neg \square \Theta, A$ by $(\rightarrow \square)_{\mathrm{S} 4.2}$. So, $\square \Gamma \subseteq \mathrm{a}(v), \square \neg \square \Theta \subseteq \mathrm{s}(v), A \in \mathrm{s}(v)$ and $\mathrm{a}(v) \cup \mathrm{s}(v) \subseteq \mathrm{Sf}(\square \Gamma \cup \square \neg \square \Theta \cup$ $\{A\}$ ) for some downward saturated sequent $v$ by Proposition 3.2. We claim that this $v$ is the required one, namely, $v \in W$ and $u R v$. Since $u R_{\mathrm{S} 4} v$ and $u R_{\mathrm{S} 4.2}^{\prime} v$ follow from $\square \Gamma \subseteq \mathrm{a}(v)$ and $\square \neg \square \Theta \subseteq \mathrm{s}(v)$ respectively, it is left to check the three properties $W(v), u Q v$, and $v R_{\mathrm{S} 4.2}^{\prime} u$.

First, let's show $W(v)$. So suppose $\square B \in \operatorname{InSf}(\mathrm{a}(v) \cup \mathrm{s}(v))$. Since $\mathrm{a}(v) \cup \mathrm{s}(v) \subseteq \operatorname{Sf}(\square \Gamma \cup \square \neg \square \Theta \cup\{A\}) \subseteq \operatorname{Sf}(\mathrm{a}(u) \cup \mathrm{s}(u))$, it follows $\square B \in$ $\operatorname{InSf}(\mathrm{a}(u) \cup \mathrm{s}(u))$, so $\square \neg \square B \in \mathrm{a}(u) \cup \mathrm{s}(u)$ by $W(u)$, and so $\square \neg \square B \in$ $\mathrm{a}(v) \cup \mathrm{s}(v)$ by $u R_{\mathrm{S} 4} v$ and $u R_{\mathrm{S} 4.2}^{\prime} v$.

Next, $u Q v$ is shown. Suppose $\square B \in \mathrm{a}(v)$. Since $\square B \in \mathrm{a}(v) \subseteq \operatorname{Sf}(\square \Gamma \cup$ $\square \neg \square \Theta \cup\{A\})$ and $\square \neg \square \Theta \subseteq \mathrm{s}(v)$, it follows $\square B \in \square \Gamma \cup \operatorname{Sf}(\Gamma \cup \square \Theta \cup\{A\}) \subseteq$ $\square \Gamma \cup \operatorname{InSf}(\mathrm{a}(u) \cup \mathrm{s}(u))$. So either $\square B \in \square \Gamma$ or $\square B \in \operatorname{InSf}(\mathrm{a}(u) \cup \mathrm{s}(u))$. In the former case, $\square B \in \mathrm{a}(u)$ since $B \in \Gamma$. In the latter case, $\square \neg \square B \in \mathrm{a}(u) \cup \mathrm{s}(u)$ by $W(u)$. But $\square \neg \square B \notin \mathrm{a}(u)$ by $u R_{\mathrm{S} 4} v, \square B \in \mathrm{a}(v)$ and Remark 3.6. Hence $\square \neg \square B \in \mathrm{s}(u)$.

Lastly, let's show $v R_{\mathrm{S} 4.2}^{\prime} u$. So suppose $\square \neg \square B \in \mathrm{s}(v)$. Since $\square \neg \square B \in$ $\mathrm{s}(v) \subseteq \operatorname{Sf}(\mathrm{a}(u) \cup \mathrm{s}(u))$, it follows $\square B \in \operatorname{InSf}(\mathrm{a}(u) \cup \mathrm{s}(u))$, and so $\square \neg \square B \in$ $\mathrm{a}(u) \cup \mathrm{s}(u)$ by $W(u)$. If $\square \neg \square B$ were in $\mathrm{a}(u)$, it would follow $\square \neg \square B \in \mathrm{a}(v)$ by $u R_{\mathrm{S} 4} v$, which is a contradiction; hence $\square \neg \square B \in \mathrm{s}(u)$.

Thanks to Proposition 3.10 as well as (3.1-a)-(3.1-e), the following proposition is easily shown by induction on the construction of formulas.

Proposition 3.11. In the canonical Kripke model $\langle W, R, V\rangle$, for every 
$u \in W$ and every $A$, if $A \in \mathrm{a}(u)$ then $u \models A$, while if $A \in \mathrm{s}(u)$ then $u \not \models A$, where $=i$ is the satisfaction relation derived from $\langle W, R, V\rangle$.

Now, to show the contraposition of Lemma 2.4, suppose that $\Gamma \rightarrow \Theta$ is GS4.2- $2^{-}$unprovable. By Proposition 3.9, $\Gamma \subseteq \mathrm{a}(u)$ and $\Theta \subseteq \mathrm{s}(u)$ for some $u \in W$. With regard to this $u$ and the canonical Kripke model $\langle W, R, V\rangle$, $u \not \models \wedge \Gamma \supset \bigvee \Theta$, since $A \in \Gamma$ implies $u \models A$, while $A \in \Theta$ implies $u \not \models A$ by Proposition 3.11. Moreover, this model is that for S4.2, namely, the accessibility relation $R$ on $W$ is reflexive, transitive and convergent by Proposition 3.5 and Corollary 3.8. Hence $\wedge \Gamma \supset \bigvee \Theta$ is unprovable in S4.2, and so $\Gamma \rightarrow \Theta$ is GS4.2-unprovable. This ends the proof of Lemma 2.4.

\section{Appendix}

The author has published [4] recently, in which some logical inference rules in sequent calculi for the modal logics are characterized semantically in a rather general setting. We will explain briefly our rule $(\rightarrow \square)_{\mathrm{S} 4.2}$ in that context.

Let GL be a sequent calculus that has $A \rightarrow A$ as an initial sequent for every $A$, and has all the structural and logical rules of propositional LK except the cut-rule as inference rules.

Definition 4.1 ([4, Definition 1.1]). A sequent $\Gamma \rightarrow \Theta$ is analytically saturated in GL, iff the following properties hold:

(4.1-a) $\Gamma \rightarrow \Theta$ is GL-unprovable.

(4.1-b) Suppose $A \in \operatorname{Sf}(\Gamma \cup \Theta)$. If $A, \Gamma \rightarrow \Theta$ is GL-unprovable, then $A \in \Gamma$; while if $\Gamma \rightarrow \Theta, A$ is GL-unprovable, then $A \in \Theta$.

If GL has $(\square \rightarrow)$ as an inference rule, analytical saturation implies downward saturation, provided that GS4.2- $2^{-}$unprovability in Definition 3.1 of the latter is replaced with GL-unprovability.

Definition 4.2 ([4, Definition 1.5]). An inference is admissible in GL, iff either some of the upper sequents of the inference is GL-unprovable, or the lower one is GL-provable.

Then, the inference rule $(\rightarrow \square)_{\mathrm{S} 4.2}$ is characterized as below (Proposition 4.3). But, due to lack of the rule $(\square \rightarrow)$ in GL, definition of the relation $Q$ in Definition 3.3(2) must be modified as follows; in spite of this modification, discussion in the previous section keeps valid after slight alterations: 
- $u Q v$, iff $\square B \in \mathrm{a}(v)$ implies either $\square B \in \mathrm{a}(u)$ or $\square \neg \square B \in \mathrm{a}(u) \cup \mathrm{s}(u)$ for every $B$.

Proposition 4.3. For a sequent calculus $\mathrm{GL}$ with the inference rule (cut) ${ }_{\mathrm{K} 5}$, the following equivalence holds for every $A$, where $W_{\mathrm{GL}}^{*}$ denotes the set of all the analytically saturated sequents $u$ 's in GL that satisfy the property $W(u)$ : The inference $(\rightarrow \square)_{\mathrm{S} 4.2}$ is admissible in GL for every $\Gamma$ and $\Theta$, iff for every $u \in W_{\mathrm{GL}}^{*}, \square A \in \mathrm{s}(u)$ implies $A \in \mathrm{s}(v)$ for some $v \in W_{\mathrm{GL}}^{*}$ such that $u R v$.

The proof of the 'only if' part is almost the same as that of Proposition 3.10, while that of the 'if' part is straightforward.

\section{References}

[1] M. Fitting, Subformula results in some propositional modal logics, Studia Logica 37 (1978), pp. 387-391.

[2] G. E. Hughes and M. J. Cresswell, A New Introduction to Modal Logic, Routledge, London and New York (1996).

[3] M. Takano, A modified subformula property for the modal logics $K 5$ and K5D, Bulletin of the Section of Logic 30 (2001), pp. 115-122.

[4] M. Takano, A semantical analysis of cut-free calculi for modal logics, Reports on Mathematical Logic 53 (2018), pp. 43-65.

[5] G. Takeuti, Proof Theory, Second Edition (Studies in Logic and the Foundations of Mathematics 81), North-Holland, Amsterdam (1987).

Professor Emeritus

Niigata University

Niigata 950-2181, Japan

e-mail: takano@emeritus.niigata-u.ac.jp 